\title{
Dark matter, lepton and baryon number, and left-right symmetric theories
}

\author{
Sudhanwa Patra',* \\ ${ }^{1}$ Center of Excellence in Theoretical and Mathematical Sciences, \\ Siksha 'O' Anusandhan University, Bhubaneswar-751030, India
}

\begin{abstract}
A lepto-baryonic left-right symmetric theory is considered along with pointing out stable dark matter candidates whose stability is ensured automatically where leptons and baryons are defined as local gauge symmetries. These theories are generally anomalous and the possible gauge anomaly free solutions for these theories are presented. It is found that the neutral component of fermion triplets can be a viable dark matter candidate originally introduced for gauge anomaly cancellation. The other dark matter possibilities within this lepto-baryonic left-right symmetric theory are also presented.
\end{abstract}

\section{INTRODUCTION}

Some of the unsolved puzzles of the Universe like tiny neutrino masses, matter-antimatter asymmetry, dark matter and dark energy, and coupling unification of three fundamental interactions have brought the realization that, the Standard Model (SM) of particle physics is unlikely to be the final theory and needs to be extended beyond its boundaries. Dark matter is the one such fascinating problem of modern cosmology contributing to $26.8 \%$ of the total energy budget in our Universe [1]. Although we have indirect gravitational evidence of dark matter from the galaxy rotation curve, gravitational lensing, and large-scale structure of the Universe, direct detection still remains a mystery. It is highly desirable to identify the particle nature of dark matter as well its other properties. The only information about dark matter-at present- is its relic abundance which is given by $\Omega_{\mathrm{DM}} h^{2}=0.119$ [1] whereas SM of particle physics cannot accommodate any candidate for dark matter. This motivates the particle physics community to propose a beyond SM framework capable of offering numerous possibilities of new physics which need to be explored with their experimental verifications. We aim here to present a beyond SM framework- a different class of left-right theories-to list the successful dark matter candidates where the stability of dark matter arises naturally.

Among all the possibilities, the left-right symmetric models based on the gauge group $S U(2)_{L} \times S U(2)_{R} \times$ $U(1)_{B-L} \times S U(3)_{C}$ [2 9] seems quite promising and is picked often for explaining the origin of neutrino masses and parity violation at the electroweak scale. The underlying mechanisms for originating light neutrino masses in these manifest LRSMs are the type-I plus typeII [6, 7, 10] seesaw mechanisms where the parity and $S U(2)_{R}$ gauge symmetry are spontaneously broken at the same scale. Apart from this, when left-right symmetry breaks at a few $\mathrm{TeV}$, it delivers rich collider phenomenology including the recent diboson excess reported by ATLAS and some low-energy processes like neutrinoless double beta decay which will confirm the lepton number violation in nature if experimentally observed. A

*Electronic address: sudha.astro@gmail.com good number of attempts have been made to address the issue of dark matter in these manifest left-right symmetric models as well $[11,12]^{1}$.

However, a different class of left-right symmetric model is considered here in which the leptons and baryons are defined as local gauge symmetries. This class of left-right symmetric model is termed "the lepto-baryonic left-right symmetric model (LB-LRSM)" and is based on the gauge group $S U(2)_{L} \times S U(2)_{R} \times U(1)_{B} \times U(1)_{L} \times S U(3)_{C} 27-$ 29] ${ }^{2}$. The rank of this LB-LRSM being six, it cannot be embedded in a known grand unified theory (GUT) model like $S U(5)$ or $S O(10)$ GUT. Any theory which can be embedded in GUT is constrained by the proton decay constraints to have the cut-off scale $>10^{15.6} \mathrm{GeV}$ - Interestingly this limit is not applicable to LB-LRSM and thus, the unification scale can be lower significantly which might lead to striking signatures.

In minimal left-right symmetric models the relic density and indirect detection including Sommerfeld enhancement push the dark matter mass beyond a few $\mathrm{TeV}$ making it unsuitable for a LHC probe. But good enough from a collider prospective, in LB-LRSM, the singlet fermion dark matter lies at a low scale, which is studied in a recent work [29]. However, this paper focuses on LB-LRSM and scrutinizes all dark matter candidates which can be accommodated in it successfully. The plan of the paper is as follows. In Sec. II, the LB-LRSM with one-stage and two-stage breaking to the SM gauge theory is studied. In Sec. III, all viable dark matter candidates that can arise naturally in LB-LRSM are discussed. The result is summarized towards the end, and the interactions for LB-LRSM are provided in the Appendix.

\footnotetext{
1 See some important studies in $\mathrm{TeV}$ scale left-right symmetric models in connection to LHC studies, Neutrinoless double beta decay and lepton flavor violation [13-26].

2 The gauge theory for baryons and lepton has been proposed in Ref. 30.
} 


\section{LEPTO-BARYONIC LEFT-RIGHT MODELS}

The lepto-baryonic left-right symmetric models (LBLRSM) are based on the basic gauge group [27 29]

$$
\mathcal{G}_{L R}^{L B} \equiv S U(2)_{L} \times S U(2)_{R} \times U(1)_{B} \times U(1)_{L} .
$$

Here we omit the $S U(3)_{C}$ structure for simplicity. The leptons, and baryons are defined as separate local gauge symmetries of the theory. The electric charge is defined as

$$
Q=T_{3 L}+T_{3 R}+\frac{B-L}{2} .
$$

Under the LB-LRSM gauge group, the usual quarks and leptons transform as

$$
\begin{aligned}
& q_{L}=\left(\begin{array}{c}
u_{L} \\
d_{L}
\end{array}\right) \equiv[2,1,1 / 3,0], q_{R}=\left(\begin{array}{c}
u_{R} \\
d_{R}
\end{array}\right) \equiv[1,2,1 / 3,0], \\
& \ell_{L}=\left(\begin{array}{c}
\nu_{L} \\
e_{L}
\end{array}\right) \equiv[2,1,0,1], \quad \ell_{R}=\left(\begin{array}{c}
\nu_{R} \\
e_{R}
\end{array}\right) \equiv[1,2,0,1] .
\end{aligned}
$$

It is found that these leptonic and baryonic currents are quantum mechanically anomalous. To have a consistent anomaly-free left-right symmetric theory one needs to introduce additional fermion degrees of freedom having nonzero lepton and baryon charges. There are many ways one can construct an anomaly-free theory of LB-LRSM which has been pointed out in the Appendix. A simple extension by additional fermion triplets $\Sigma_{L} \oplus \Sigma_{R}$ is considered to serve the purpose,

$$
\begin{aligned}
\Sigma_{L} & =\left(\begin{array}{cc}
\Sigma_{L, R}^{0} & \sqrt{2} \Sigma_{L, R}^{+} \\
\sqrt{2} \Sigma_{L, R}^{-} & -\Sigma_{L, R}^{0}
\end{array}\right) \equiv[3,1,-3 / 4,-3 / 4] \\
\Sigma_{R} & =\left(\begin{array}{cc}
\Sigma_{R}^{0} & \sqrt{2} \Sigma_{R}^{+} \\
\sqrt{2} \Sigma_{R}^{-} & -\Sigma_{R}^{0}
\end{array}\right) \equiv[1,3,-3 / 4,-3 / 4]
\end{aligned}
$$

The motivation behind introducing fermion triplets is twofold:

- It cancels a nontrivial gauge anomaly making the lepto-baryonic Left-Right symmetric models anomaly-free theories.

- The neutral component of these fermion triplets can be stable cold dark matter where the stability is ensured automatically.

The spontaneous symmetry breaking of LB-LRSM can be done in different ways leading to interesting phenomenology. However, in this work we consider only two-stage and one-stage breaking of LB-LRSM to the SM gauge group discussed in the following subsections.

\section{A. Two-stage symmetry breaking}

The spontaneous symmetry breaking of the leptobaryonic left-right symmetric models (LB-LRSM), in which the symmetry breaking of LB-LRSM to the SM gauge group occurs via two-step breaking, is presented below:

$$
\mathcal{G}_{L R}^{L B} \stackrel{\left\langle S_{L B}\right\rangle}{\longrightarrow} \mathcal{G}_{L R} \stackrel{\left\langle\Delta_{R}\right\rangle}{\longrightarrow} \mathcal{G}_{S M} \stackrel{\langle\phi\rangle}{\longrightarrow} U(1)_{\mathrm{em}}
$$

where

$$
\begin{aligned}
& \mathcal{G}_{L R}^{L B} \equiv S U(2)_{L} \times S U(2)_{R} \times U(1)_{B} \times U(1)_{L}, \\
& \mathcal{G}_{L R} \equiv S U(2)_{L} \times S U(2)_{R} \times U(1)_{B-L}, \\
& \mathcal{G}_{S M} \equiv S U(2)_{L} \times U(1)_{Y},
\end{aligned}
$$

omitting the $S U(3)_{C}$ structure for simplicity. Alternatively, the symmetry-breaking pattern for two-stage breaking of LB-LRSM can also be understood as follows

$$
\begin{aligned}
& S U(2)_{L} \times S U(2)_{R} \times U(1)_{B} \times U(1)_{L} \otimes \mathcal{P} \\
& \left\{T_{L}, T_{3 L}\right\} \quad\left\{T_{R}, T_{3 R}\right\} \quad \mathrm{B} \quad \mathrm{L} \\
& g_{L}\left(M_{L B}\right) \quad g_{R}\left(M_{L B}\right) \\
& \downarrow\left\langle S_{L B}(1,1,3 / 2,3 / 2)\right\rangle \quad\left\{M_{S}, M_{Z_{B}}, M_{Z_{\ell}}\right\} \\
& S U(2)_{L} \times S U(2)_{R} \times U(1)_{B-L} \\
& \left\{T_{L}, T_{3 L}\right\} \quad\left\{T_{R}, T_{3 R}\right\} \quad \mathrm{B}-\mathrm{L} \quad Q=T_{3 L}+T_{3 R}+\frac{B-L}{2} \\
& g_{L}\left(M_{R}\right) \\
& \underbrace{g_{R}\left(M_{R}\right) g_{B L}}_{\downarrow\left\langle\Delta_{R}(1,3,0,-2)\right\rangle} \\
& \begin{array}{ccc}
S U(2)_{L} & \times U(1)_{Y} \\
\left\{T_{L}, T_{3 L}\right\} & Y
\end{array} \\
& \underbrace{g \equiv g_{L} \quad g^{\prime}}_{\downarrow\left\langle\phi\left(1, \frac{1}{2}\right)\right\rangle} \\
& U(1)_{\mathrm{em}} \\
& \text { e } \\
& Q=T_{3 L}+Y
\end{aligned}
$$


Additional discrete left-right symmetry is imposed ensuring equality between $S U(2)_{L}$ and $S U(2)_{R}$ gauge couplings, i.e, $g_{L}=g_{R}$ by either parity $\mathcal{P}$ or chargeconjugation $\mathcal{C}$ symmetry.

The phenomenologically interesting two-stage breaking of the LB-LRSM can be understood in following ways:

- At first, the spontaneous symmetry breaking of $U(1)_{B} \times U(1)_{L}$ to $U(1)_{B-L}$ happens via a scalar $S_{L B}(1,1,3 / 2,3 / 2)$ singlet under $S U(2)_{L, R}$. The inclusion of the scalar not only does the job of spontaneous symmetry breaking but also gives masses to extra fermion triplets though

$$
\lambda_{\Sigma} \operatorname{Tr}\left(\Sigma_{L} \Sigma_{L}+\Sigma_{R} \Sigma_{R}\right) S_{L B}
$$

- The second stage of symmetry breaking is done as in the usual LR symmetric model where the breaking of $S U(2)_{R} \times U(1)_{B-L}$ to $U(1)_{Y}$ is achieved by scalar triplets $\Delta_{L}(3,1,-2)+\Delta_{R}(1,3,-2)$. However, this stage of symmetry breaking can be implemented via scalar doublets $H_{L}(2,1,-1)+H_{R}(1,2,-1)$.

The advantage of two-stage symmetry breaking is that one can make an analogy with the minimal left-right symmetric model and its low scale phenomenology. The LBLRSM with scalar triplets, and scalar bidoublet, along with scalar singlet are

$$
\begin{aligned}
& \Phi=\left(\begin{array}{cc}
\phi_{1}^{0} & \phi_{2}^{+} \\
\phi_{1}^{-} & \phi_{2}^{0}
\end{array}\right) \equiv[2,2,0,0], \\
& \Delta_{L} \equiv\left(\begin{array}{cc}
\delta_{L}^{+} / \sqrt{2} & \delta_{L}^{++} \\
\delta_{L}^{0} & -\delta_{L}^{+} / \sqrt{2}
\end{array}\right) \equiv[3,1,0,-2], \\
& \Delta_{R} \equiv\left(\begin{array}{cc}
\delta_{R}^{+} / \sqrt{2} & \delta_{R}^{++} \\
\delta_{R}^{0} & -\delta_{R}^{+} / \sqrt{2}
\end{array}\right) \equiv[1,3,0,-2], \\
& S_{L B} \equiv[1,1,3 / 2,3 / 2]
\end{aligned}
$$

With scalar triplets $\Delta_{L} \oplus \Delta_{R}$, the light neutrino masses are governed by type-I plus type-II seesaw mechanisms.
The neural lepton mass matrix is given by

$$
M_{\nu}=\left(\begin{array}{ll}
M_{L} & M_{D} \\
M_{D}^{T} & M_{R}
\end{array}\right)
$$

where the Majorana mass matrix for left-handed (righthanded) neutrinos is $M_{L}=f_{L}\left\langle\Delta_{L}\right\rangle=f v_{L}\left(M_{R}=\right.$ $f_{R}\left\langle\Delta_{R}\right\rangle=f v_{R}$ while the Dirac neutrino mass matrix connecting light-heavy neutrinos is $m_{D}=Y_{3} v_{1}+Y_{4} v_{2}$. This results in a mass formula for light as well as heavy neutrinos as

$$
m_{\nu}=M_{L}-m_{D} M_{R}^{-1} m_{D}^{T}=m_{\nu}^{I I}+m_{\nu}^{I},
$$

The consequences of the LRSM seesaw mechanism to neutrinoless double beta decay, LFV and collider studies have been explored recently. This will be applicable to LB-LRSM if the symmetry breaking happens at a few $\mathrm{TeV}$ scale with an additional signature which might discriminate between minimal LRSM and LB-LRSM in the future.

\section{B. One-stage symmetry breaking}

The symmetry-breaking chain of LB-LRSM, in which LB-LRSM to SM symmetry breaking occurs in single stage, is given by

$$
\mathcal{G}_{L R}^{L B} \stackrel{\left\langle H_{R}\right\rangle,\left\langle S_{L B}\right\rangle}{\longrightarrow} \mathcal{G}_{S M} \stackrel{\langle\Phi\rangle,\left\langle H_{L}\right\rangle}{\longrightarrow} U(1)_{\mathrm{em}}
$$

where,

$$
\begin{aligned}
& \mathcal{G}_{L R}^{L B} \equiv S U(2)_{L} \times S U(2)_{R} \times U(1)_{B} \times U(1)_{L}, \\
& \mathcal{G}_{S M} \equiv S U(2)_{L} \times U(1)_{Y},
\end{aligned}
$$

omitting the $S U(3)_{C}$ structure for simplicity. This can also be explained in greater detail as follows:

$$
\begin{aligned}
& S U(2)_{L} \times S U(2)_{R} \times U(1)_{B} \times U(1)_{L} \\
& \left\{T_{L}, T_{3 L}\right\} \quad\left\{T_{R}, T_{3 R}\right\} \quad \mathrm{B} \quad \mathrm{L} \\
& g_{L}\left(M_{L B}\right) \underbrace{g_{R}\left(M_{L B}\right) \quad g_{B} \quad g_{\ell}}_{\downarrow\left\langle S_{L B}\right\rangle,\left\langle H_{R}\right\rangle}\left\{M_{\left.Z_{B}, M_{Z_{\ell}}, M_{S}, M_{H}, \cdots\right\}}\right. \\
& S U(2)_{L} \times U(1)_{Y} \\
& \left\{T_{L}, T_{3 L}\right\} \quad Y \\
& \underbrace{g \equiv g_{L} g^{\prime}}_{\downarrow\left\langle\phi\left(1, \frac{1}{2}\right)\right\rangle} \\
& U(1)_{\mathrm{em}} \\
& e \\
& Q=T_{3 L}+Y
\end{aligned}
$$

Implementation of spontaneous symmetry breaking can be done via Higgs doublets with $B-L \neq 0$ plus lepto- baryonic $S U(2)$-singlet scalar without the need for a 
scalar bidoublet with zero $B$ and $L$ charges. Here the fermion masses and mixing can be explained by introducing vectorlike fermions via a universal seesaw mechanism. With the introduction of scalar bidoublet, the symmetry breaking can be achieved either with scalar doublets or scalar triplets plus a lepto-baryonic $S U(2)$-singlet scalar. The role of the lepto-baryonic $S U(2)$-singlet scalar is to give the Majorana mass for lepto-baryons needed for gauge anomaly cancellation. In the present work, the symmetry breaking of $\mathcal{G}_{L R}^{L B} \rightarrow \mathcal{G}_{S M}$ as well as for $\mathcal{G}_{S M} \rightarrow U(1)_{\text {em }}$ is done with $H_{R}, H_{L}, S_{L B}$, and $\phi$ contained in a bidoublet scalar.

We present below one such gauge anomaly-free LBLRSM comprising of fermions as follows

$$
\begin{aligned}
& q_{L} \equiv[2,1,1 / 3,0], \quad q_{R} \equiv[1,2,1 / 3,0] \\
& \ell_{L} \equiv[2,1,0,1], \quad \ell_{R} \equiv[1,2,0,1] \\
& \Sigma_{L} \equiv[3,1,-3 / 4,-3 / 4], \quad \Sigma_{R} \equiv[1,3,-3 / 4,-3 / 4]
\end{aligned}
$$

while the scalar sector is comprising

$$
\begin{aligned}
& \Phi=\left(\begin{array}{cc}
\phi_{1}^{0} & \phi_{2}^{+} \\
\phi_{1}^{-} & \phi_{2}^{0}
\end{array}\right) \equiv[2,2,0,0], \\
& H_{L}=\left(\begin{array}{c}
h_{L}^{+} \\
h_{L}^{0}
\end{array}\right) \equiv[2,1,3 / 4,-1 / 4], \\
& H_{R}=\left(\begin{array}{c}
h_{R}^{+} \\
h_{R}^{0}
\end{array}\right) \equiv[1,2,3 / 4,-1 / 4], \\
& S_{L B} \equiv[1,1,3 / 2,3 / 2]
\end{aligned}
$$

The spontaneous symmetry breaking of LB-LRSM $\mathcal{G}_{L R}^{L B}$ to $\mathrm{SM}$ is achieved by assigning non-zero vevs to $H_{R}(1,2,3 / 4,-1 / 4)$ and $S_{L B}(1,1,3 / 2,3 / 2)$. Later, the SM gauge group breaks down to $U(1)_{\mathrm{em}}$ by the SM Higgs doublet contained in the LR bidoublet as $\Phi(2,2,0,0)$. The left-handed counterpart $H_{L}(2,1,3 / 4,-1 / 4)$ is needed from the left-right symmetry. The phenomenology of this kind of LB-LRSM is recently studied in Ref. 28] in the context of light neutrino masses via the type-III seesaw mechanism. The details of the interaction Lagrangian is presented in Appendix.

However, one can assign different $B$ and $L$ charges for $H_{L} \oplus H_{R}$ such that the electric charge for individual components $h_{L, R}^{+}$and $h_{L, R}^{0}$ should be +1 and 0 , respectively, satisfying the electric charge relation

$$
Q=T_{3 L}+T_{3 R}+\frac{B-L}{2}
$$

where, $T_{3 L}\left(T_{3 R}\right)$ is the third component of the isospin generator for gauge groups $S U(2)_{L}\left(S U(2)_{R}\right)$. The reason for choosing $B=3 / 4$ and $L=-1 / 4$ for $H_{L, R}$ is to allow the $\ell_{L, R} \Sigma_{L, R} H_{L, R}$ terms for implementation of the type-III seesaw.

The sub-eV scale of light neutrinos can also be explained via the Dirac neutrino mass $M_{\nu}^{D} \equiv$ $\frac{1}{\sqrt{2}}\left(Y_{3} v_{1}+Y_{4} v_{2}\right)$ by suitably adjusting the Yukawa couplings. The key point to note here is that one can assign different $B$ and $L$ charges for $H_{L, R}$ consistent with the above charge relation. For example, $H_{L}(2,1,1 / 2,-1 / 2)$,
$H_{R}(1,2,1 / 2,-1 / 2)$ is a viable choice for spontaneous symmetry breaking of LB-LRSM to SM. Similarly, the spontaneous symmetry breaking of LB-LRSM to SM can be implemented with scalar triplets $\Delta_{L}(3,1,1,-1) \oplus$ $\Delta_{R}(1,3,1,-1)$. In those classes of LB-LRSM theories, the neutral component of the fermion triplets, originally introduced for gauge anomaly cancellation, can be a viable dark matter candidate where stability is ensured automatically. Since both Higgs doublets as well as Higgs triplets do not couple to leptons, there is no Majorana mass term for left-handed as well as right-handed neutrinos, and thus, type-I plus type-II seesaw terms are absent. Then, the mechanism of the light neutrino masses can be understood via extended seesaw mechanism by adding singlet fermions which is beyond the scope of this work.

\section{STABLE DARK MATTER CANDIDATES}

The prime aim here is to present viable dark matter candidates within lepto-baryonic left-right symmetric theories.

\section{A. Lepto-Baryons as dark matter}

From previous discussions, it is clear that we need lepto-baryons [which transform under $S U(2)$ as triplets] $\Sigma_{L}(3,1,-3 / 4,-3 / 4) \oplus \Sigma_{R}(1,3,-3 / 4,-3 / 4)$, as a simple example, to make the LB-LRSM anomaly-free gauge theory. The key point to note here is that the neutral component of these lepto-baryons needed for anomaly cancellation can be a stable dark matter candidate where stability of the dark matter is ensured automatically. The relevant interaction Lagrangian for lepto-baryonic dark matter within LB-LRSM is given by

$$
\begin{aligned}
\mathcal{L}_{\Sigma}= & -\frac{y_{\Sigma}}{2} S_{L B} \operatorname{Tr}\left(\Sigma_{L} \Sigma_{L}+\Sigma_{R} \Sigma_{R}\right) \\
& -e A_{\mu} \overline{\Sigma_{L, R}^{+}} \gamma^{\mu} \Sigma_{L, R}^{+}-g_{L, R} c_{W} Z_{L, R_{\mu}} \overline{\Sigma_{L, R}^{+}} \gamma^{\mu} \Sigma_{L, R}^{+} \\
- & g_{L, R}\left(W_{L, R_{\mu}}^{+} \overline{\Sigma_{L, R}^{+}} \gamma^{\mu} \Sigma_{L, R}^{0}+\text { h.c. }\right) \\
- & \frac{3}{4} g_{B} Z_{B_{\mu}}\left(\overline{\Sigma_{L, R}^{+}} \gamma^{\mu} \gamma^{5} \Sigma_{L, R}^{+}+\frac{1}{4} \Sigma_{L, R}^{0} \gamma^{\mu} \gamma^{5} \Sigma_{L, R}^{0}\right) \\
- & \frac{3}{4} g_{\ell} Z_{\ell_{\mu}}\left(\overline{\Sigma_{L, R}^{+}} \gamma^{\mu} \gamma^{5} \Sigma_{L, R}^{+}+\frac{1}{4} \Sigma_{L, R}^{0} \gamma^{\mu} \gamma^{5} \Sigma_{L, R}^{0}\right)(11)
\end{aligned}
$$

The radiative corrections give mass splitting between neutral and charged components of lepto-baryonic fermion triplets. For left-handed fermion triplets, the mass splitting is $166 \mathrm{MeV}$, whereas the mass splitting among right-handed triplets will depend upon the mass of the heavy gauge bosons $W_{R}, Z_{R}$ involved in the loop. The dark matter phenomenology for triplets will differ from Refs. [11, 12] due to additional coannihilation effects from $Z_{B, \ell}$ gauge boson-mediated diagrams. Thus, in LB-LRSM, the possible annihilation channels are as follows: 
- The scalar portal annihilation channels are

$$
\begin{aligned}
\Sigma_{1} \Sigma_{1} \rightarrow & H_{i}^{*} \rightarrow \bar{q}_{i} q_{i}, \bar{e}_{i} e_{i}, \bar{\nu}_{i} \nu_{i}, \\
& W W, Z Z, Z_{1,2}^{\prime} Z_{1,2}^{\prime}, W_{R}^{+} W_{R}^{-}, H^{ \pm \pm} H^{\mp \mp},
\end{aligned}
$$

Here, we denote $H_{i}$ and $H^{\mp \mp}$ as the heavy scalars and $Z_{1,2}^{\prime}$ as extra neutral gauge bosons. We can ignore most of these channels by assuming the small mixing between the singlet scalar $S_{L B}$ and other Higgs scalars.

- The $Z^{\prime}$ portal annihilation channels are

$$
\Sigma_{1} \Sigma_{1} \rightarrow Z_{i}^{\prime} \rightarrow \bar{q}_{i} q_{i}, \bar{e}_{i} e_{i}, \bar{\nu}_{i} \nu_{i}
$$

- The important t-channel processes contributing to relic density calculation are

$\Sigma_{1} \Sigma_{1} \rightarrow H_{i} Z_{j}^{\prime}, Z_{i}^{\prime} Z_{j}^{\prime}, H_{i} H_{j}, W^{+} W^{-}, W_{R}^{+} W_{R}^{-}, H^{ \pm} W^{\mp}$.

Following the dark matter phenomenology discussed in Refs. 11, 12 and the standard calculation for Majorana dark matter via the $Z_{B, \ell}$-portal, one can explain PLANCK-WMAP 31 with a few TeV dark matter masses. The detailed dark matter phenomenology is beyond the scope of this paper.

\section{B. Minimal Left-Right dark matter possibilities}

Here the possibilities of having stable TeV-scale dark matter in lepto-baryonic left-right models is explained in a manner followed in a recent work 11] where the stability of the dark matter is either ensured automatically because of a high $S U(2)$ dimension or due to the remnant discrete symmetry arising after spontaneous breaking of the $U(1)_{B-L}$ gauge group. The goal of this work is to only provide all stable dark matter components, and omit a detailed discussion, as the same has been discussed in [11, 12].

At first the scalar singlet $S_{L B}(1,1,3 / 2,3 / 2)$ under $S U(2)_{L, R}$ breaks lepto-baryonic left-right theories $S U(2)_{L} \times S U(2)_{R} \times U(1)_{B} \times U(1)_{L}$ to conventional left-right models $S U(2)_{L} \times S U(2)_{R} \times U(1)_{B-L}$. Later the spontaneous breaking of LRSM to SM and further breaking of SM to a low-energy theory is governed by usual scalar bidoublet $\Phi(2,2,0)$ and scalar triplets $\Delta_{R}(1,3,2), \Delta_{L}(3,1,2)$. When the symmetry breaking $S U(2)_{R} \times U(1)_{B-L}$ occurs at conventional leftright breaking scale, there is a remnant discrete symmetry

$$
\mathcal{Z}_{2} \simeq(-1)^{B-L} \text { or }(-1)^{3(B-L)}[11,12 \text {. }
$$

Under this remnant discrete symmetry, all quarks and leptons are odd while all bosons are even. Thus, one can add a new dark matter multiplet which is a fermion (scalar) transforming under remnant discrete symmetry $\mathcal{Z}_{2}$ as even (odd). Also, the multiplets having a high $S U(2)$ dimension can act as a dark matter candidate if the tree level decay is forbidden at the renormalizable level. Thus, the different dark matter multiplets are presented below where the new dark matter particle is accidentally stable because the high $S U(2)$ dimensionality forbids renormalizable couplings that could lead to decay, or the stability of dark matter is ensured due to their quantum numbers under the remnant discrete symmetry $\mathbb{Z}_{2} \subset U(1)_{B-L}$ arising after spontaneous breaking of the left-right gauge group via the scalar triplets $\Delta_{L, R}$.

\section{Fermionic dark matter :}

$$
\chi_{L} \sim(\mathbf{2 n}+\mathbf{1}, \mathbf{1}, 0), \quad \chi_{R} \sim(\mathbf{1}, \mathbf{2 n}+\mathbf{1}, 0)
$$

where $n \in \mathbb{N}$ and this results in two component dark matter with common Majorana mass $M$ due to LR exchange symmetry. The condition, $B-L=0$ for these fermionic dark matter multiplets $(\mathbf{2 n}+\mathbf{1}, \mathbf{1}, 0)+(\mathbf{1}, \mathbf{2 n}+\mathbf{1}, 0)$ can be contained in a higher multiplet of LB-LRSM either with the same or zero $B$ and $L$ charges. This possibility may give different dark matter phenomenology unlike in minimal left-right dark matter phenomenology [11, 12].

Scalar dark matter : For dark matter multiplets whose stability is ensured automatically because of higher $S U(2)$ dimension forbidding any tree level decay of dark matter,

$$
\begin{aligned}
& \chi_{L}\left(\mathbf{2} \boldsymbol{j}_{\mathbf{1}}+\mathbf{1}, \mathbf{2} \boldsymbol{j}_{\mathbf{2}}+\mathbf{1}, 0\right) \oplus \chi_{R}\left(\mathbf{2} \boldsymbol{j}_{\mathbf{2}}+\mathbf{1}, \mathbf{2} \boldsymbol{j}_{\mathbf{1}}+\mathbf{1}, 0\right) \\
& \text { or } \eta(\mathbf{2} \boldsymbol{j}+\mathbf{1}, \mathbf{2} \boldsymbol{j}+\mathbf{1}, 0),
\end{aligned}
$$

In addition, there could be other dark matter possibilities whose stability is guaranteed by the remnant discrete symmetry $\mathcal{Z}_{2}$ after spontaneous symmetry breaking of $S U(2)_{R} \times U(1)_{B-L}$ with $j, j_{1,2} \in \mathbb{N}$. The simplest example is scalar doublet dark matter

$$
H_{L}(\mathbf{2}, \mathbf{1},-1) \oplus H_{R}(\mathbf{1}, \mathbf{2},-1) .
$$

\section{Other dark matter possibilities}

Fermion Singlet Dirac dark matter : Very recently, it was pointed out in Ref. 29] that a vectorlike fermionic Dirac dark matter can be easily accommodated within LB-LRSM. The relevant interactions for this singlet fermion Dirac dark matter $\chi\left(1,1, n_{b}, n_{\ell}\right)$ with $n_{B}=n_{\ell}$ is

$$
\begin{aligned}
\mathcal{L}_{\chi}^{\text {Dirac }} & =-n_{b} g_{B} \bar{\chi} \gamma_{\mu} \chi Z_{B}^{\mu}-n_{\ell} g_{\ell} \bar{\chi} \gamma_{\mu} \chi Z_{\ell}^{\mu} \\
& -\frac{1}{3} g_{B} \bar{q} \gamma_{\mu} q Z_{B}^{\mu}-g_{\ell} \bar{\ell} \gamma_{\mu} \ell Z_{\ell}^{\mu} \\
& -M_{\chi} \bar{\chi} \chi+\cdots
\end{aligned}
$$

Fermion Singlet Majorana dark matter : Include a Majorana fermion singlet under $S U(2)_{L, R}$ and equal baryon and lepton number $\chi_{L}\left(1,1, n_{b}, n_{\ell}\right) \oplus$ $\chi_{R}\left(1,1, n_{b}, n_{\ell}\right)$ to the minimal setup for lepto-baryonic left-right symmetric theory. The relevant interactions for 
Majorana dark matter is given by

$$
\begin{aligned}
\mathcal{L}_{\chi}^{\text {Majorana }} & -\frac{3}{4} g_{B} \overline{\chi_{L}} \gamma_{\mu} \gamma^{5} \chi_{L} Z_{B}^{\mu}-\frac{3}{4} g_{\ell} \overline{\chi_{L}} \gamma_{\mu} \gamma^{5} \chi_{L} Z_{\ell}^{\mu} \\
& -\frac{3}{4} g_{B} \overline{\chi_{R}} \gamma_{\mu} \gamma^{5} \chi_{R} Z_{B}^{\mu}-\frac{3}{4} g_{\ell} \overline{\chi_{R}} \gamma_{\mu} \gamma^{5} \chi_{R} Z_{\ell}^{\mu} \\
& -\frac{1}{3} g_{B} \bar{q} \gamma_{\mu} q Z_{B}^{\mu}-g_{\ell} \bar{\ell} \gamma_{\mu} \ell Z_{\ell}^{\mu} \\
& -\lambda_{\chi} S_{L B} \overline{\chi^{c}}{ }_{L} \chi_{L}-\lambda_{\chi}^{\prime} S_{L B} \overline{\chi^{c}}{ }_{R} \chi_{R}
\end{aligned}
$$

The phenomenology of Majorana fermion dark matter via $Z^{\prime}$ portal as well as Higgs portal will be studied in a separate work.

Fermion Doublet dark matter : A few solutions for gauge anomaly cancellation within lepto-baryonic LR theories are presented in the Appendix. One of these solutions is lepto-baryons having doublet under $S U(2)_{L, R}$ with lepton and baryon number as -3 . This immediately results in $B-L=0$, and the electric charge relation

$$
Q=T_{3 L}+T_{3 R}+\frac{B-L}{2}
$$

implies the neutral component has a nonzero hypercharge. Thus, the fermionic doublet dark matter can couple to SM $Z$ and thereby is ruled out from direct detection constraints.

\section{CONCLUSION}

The lepto-baryonic Left-Right symmetric theory is addressed by defining leptons and baryons as local gauge symmetries. The one-stage and two-stage spontaneous breaking of this lepto-baryonic left-right symmetric theory to the SM gauge theory via scalar triplets and scalar doublets are studied. The viability of stable dark matter candidates as triplets, doublets and singlets (Dirac as well as Majorana fermion) in these gauge anomaly-free theories is also discussed. The detailed numerical dark matter phenomenology has been omitted for a separate work. Towards the end the Lagrangian interaction for both triplet and doublet versions of lepto-baryonic leftright symmetric theories has been provided.

\section{ACKNOWLEDGEMENT}

The author thanks Werner Rodejohann for the visiting position at the Max-Planck Institute for Kernphysik, Heidelberg, Germany where this idea was conceived. The work of SP is partially supported by the Department of Science and Technology, Govt. of India under the financial grant SB/S2/HEP-011/2013.

\section{APPENDIX: ANOMALIES IN LEFT-RIGHT THEORIES WITH $S U(2)_{L} \times S U(2)_{R} \times U(1)_{B} \times U(1)_{L}$}

We discuss below the induced gauge anomalies in the left-right symmetric model with the gauge group
$S U(2)_{L} \times S U(2)_{R} \times U(1)_{B} \times U(1)_{L}$ omitting the $S U(3)_{C}$ structure for simplicity where baryon and lepton numbers are promoted to local gauge symmetries. For completeness, we also present a complete Lagrangian for a simplified version of this class of left-right symmetric theories.

\section{A. Few gauge anomaly free solutions for lepto-baryonic Left-Right Models}

The lepto-baryonic Left-Right symmetric models with gauge group $\mathcal{G}_{L, R}^{B L} \equiv S U(2)_{L} \times S U(2)_{R} \times U(1)_{B} \times U(1)_{L^{-}}$ skipping the $S U(3)_{C}$ structure for simplicity- induces non-zero gauge anomalies in the theory,

$$
\begin{aligned}
& \mathcal{A}\left[S U(2)_{L}^{2} \times U(1)_{B}\right]=3 / 2, \\
& \mathcal{A}\left[S U(2)_{R}^{2} \times U(1)_{B}\right]=-3 / 2, \\
& \mathcal{A}\left[S U(2)_{L}^{2} \times U(1)_{L}\right]=3 / 2, \\
& \mathcal{A}\left[S U(2)_{R}^{2} \times U(1)_{L}\right]=-3 / 2,
\end{aligned}
$$

Although solutions to these gauge anomalies have been presented in Refs. 27, 29], it will be good to note them down here so that dark matter studies can be applicable to other scenarios also, if applicable, as

- The simplest way to cancel a gauge anomaly is to include the fermion triplets $\Sigma_{L} \oplus \Sigma_{R}$ as follows:

$$
\Sigma_{L}=\left(\begin{array}{cc}
\Sigma_{L, R}^{0} & \sqrt{2} \Sigma_{L, R}^{+} \\
\sqrt{2} \Sigma_{L, R}^{-} & -\Sigma_{L, R}^{0}
\end{array}\right), \quad \Sigma_{R}=\left(\begin{array}{cc}
\Sigma_{R}^{0} & \sqrt{2} \Sigma_{R}^{+} \\
\sqrt{2} \Sigma_{R}^{-} & -\Sigma_{R}^{0}
\end{array}\right)
$$

- Lepto-baryons doublet under $S U(2)_{L, R}$ and color singlet carrying nonzero baryon and lepton numbers,

$S U(3)_{C}$ singlets $\left\{\begin{aligned} \Psi_{L}^{1} & \sim(2,1,-n,-n) \\ \Psi_{R}^{1} & \sim(1,2,-n,-n),\end{aligned}\right.$

- Lepto-Baryons which are nonsinglets under both $S U(2)_{L, R}$ and triplets under $S U(3)_{C}$ with nonzero lepton and baryon numbers,

$$
S U(3)_{C} \operatorname{triplets}\left\{\begin{array}{r}
\Psi_{L}^{3} \sim(2,1,-n / 3,-n / 3) \\
\Psi_{R}^{3} \sim(1,2,-n / 3,-n / 3),
\end{array}\right.
$$

- Lepto-baryons with $N$-dimensional representation of $S U(3)_{C}$ and doublets under $S U(2)_{L, R}$ with nonzero $B$ and $L$,

$S U(3)_{C}$ N-tuplets $\left\{\begin{array}{l}\Psi_{L}^{N} \sim(2,1,-n / N,-n / N) \\ \Psi_{R}^{N} \sim(1,2,-n / N,-n / N)\end{array}\right.$

with $n$ being the number of fermion family generation which is 3 , 


\section{B. Lagrangian for lepto-baryonic Left-Right Theories}

The Lagrangian for the present lepto-baryonic leftright theories where the spontaneous symmetry breaking is achieved with scalar sector comprising of $H_{R}, H_{L}, \Phi, S_{L B}$ is given below:

$$
\mathcal{L}_{\mathrm{LR}}^{\mathrm{LB}}=\mathcal{L}_{\text {scalar }}+\mathcal{L}_{\text {Kin. }}^{\text {gauge }}+\mathcal{L}_{\mathrm{Kin} .}^{\text {fermion }}+\mathcal{L}_{\text {Yuk }}
$$

where the individual parts can be written as

$$
\begin{aligned}
\mathcal{L}_{\text {scalar }}= & \operatorname{Tr}\left[\left(\mathcal{D}_{\mu} \Phi\right)^{\dagger}\left(\mathcal{D}^{\mu} \Phi\right)\right]+\left(\mathcal{D}_{\mu} S_{B L}\right)^{\dagger}\left(\mathcal{D}^{\mu} S_{B L}\right) \\
+ & \left(\mathcal{D}_{\mu} H_{L}\right)^{\dagger}\left(\mathcal{D}^{\mu} H_{L}\right)+\left(\mathcal{D}_{\mu} H_{R}\right)^{\dagger}\left(\mathcal{D}^{\mu} H_{R}\right) \\
& -\mathcal{V}\left(\Phi, H_{L}, H_{R} S_{B L}\right)
\end{aligned}
$$

Defining $\Phi \equiv \Phi_{1}$ and $\Phi_{2}=\tau_{2} \Phi^{*} \tau_{2}$, the scalar potential can be written as follows

$$
\begin{aligned}
\mathcal{V}( & \left., H_{L}, H_{R}, S_{L B}\right)=-\sum_{i, j=1,2} \frac{\mu_{\phi i j}^{2}}{2} \operatorname{Tr}\left(\Phi_{i}^{\dagger} \Phi_{j}\right) \\
& +\sum_{i, j, k, l=1,2} \frac{\lambda_{\phi i j k l}}{4} \operatorname{Tr}\left(\Phi_{i}^{\dagger} \Phi_{j}\right) \operatorname{Tr}\left(\Phi_{k}^{\dagger} \Phi_{l}\right) \\
& +\sum_{i, j, k, l=1,2} \frac{\Lambda_{\phi i j k l}}{4} \operatorname{Tr}\left(\Phi_{i}^{\dagger} \Phi_{j} \Phi_{k}^{\dagger} \Phi_{l}\right) \\
& -\mu_{H}^{2}\left(H_{L}^{\dagger} H_{L}+H_{R}^{\dagger} H_{R}\right) \\
& +\lambda_{1}\left[\left(H_{L}^{\dagger} H_{L}\right)^{2}+\left(H_{R}^{\dagger} H_{R}\right)^{2}\right]+\lambda_{2}\left(H_{L}^{\dagger} H_{L}\right)\left(H_{R}^{\dagger} H_{R}\right) \\
& +\sum_{i, j} \beta_{i j}\left(H_{L}^{\dagger} H_{L}+H_{R}^{\dagger} H_{R}\right) \operatorname{Tr}\left(\Phi_{i}^{\dagger} \Phi_{j}\right) \\
& +\sum_{i, j} \varrho_{i j}\left[H_{L}^{\dagger} \Phi_{i} \Phi_{j}^{\dagger} H_{L}+H_{R}^{\dagger} \Phi_{i}^{\dagger} \Phi_{i} H_{R}\right] \\
& -\mu_{S}^{2} S_{L B}^{\dagger} S_{L B}+\lambda_{S}\left(S_{L B}^{\dagger} S_{L B}\right)^{2} \\
& +\lambda_{S H}\left(S_{L B}^{\dagger} S_{L B}\right)\left(H_{L}^{\dagger} H_{L}+H_{R}^{\dagger} H_{R}\right) \\
& +\sum_{i, j} \lambda_{S \phi i j}\left(S_{L B}^{\dagger} S_{L B}\right) \operatorname{Tr}\left(\Phi_{i}^{\dagger} \Phi_{j}\right) .
\end{aligned}
$$

The kinetic terms for the gauge bosons are given by

$$
\begin{aligned}
\mathcal{L}_{\text {Kin. }}^{\text {gauge }}= & -\frac{1}{4} W_{\mu \nu L} \cdot W^{\mu \nu L}-\frac{1}{4} W_{\mu \nu R} \cdot W^{\mu \nu R} \\
& -\frac{1}{4} B_{\mu \nu}^{B} B^{\mu \nu, B}-\frac{1}{4} B_{\mu \nu}^{L} B^{\mu \nu, L}
\end{aligned}
$$

while for fermions,

$$
\begin{aligned}
\mathcal{L}_{\text {Kin. }}^{\text {fermion }}= & i \overline{q_{L}} \gamma^{\mu} \mathcal{D}_{\mu} q_{L}+i \overline{q_{R}} \gamma^{\mu} \mathcal{D}_{\mu} q_{R} \\
& +i \overline{\ell_{L}} \gamma^{\mu} \mathcal{D}_{\mu} \ell_{L}+i \overline{\ell_{R}} \gamma^{\mu} \mathcal{D}_{\mu} \ell_{R} \\
& +i \overline{\Sigma_{L}} \gamma^{\mu} \mathcal{D}_{\mu} \Sigma_{L}+i \overline{\Sigma_{R}} \gamma^{\mu} \mathcal{D}_{\mu} \Sigma_{R}
\end{aligned}
$$

However we can define the respective covariant derivatives, in general, as
[1] Planck, P. A. R. Ade et al., "Planck 2013 results. XVI. Cosmological parameters," Astron. Astrophys. 571 (2014) A16, arXiv:1303.5076.

[2] R. Mohapatra and J. C. Pati, "A Natural Left-Right Symmetry," Phys.Rev. D11 (1975) 2558

[3] J. C. Pati and A. Salam, "Lepton Number as the Fourth Color," Phys.Rev. D10 (1974) 275-289.

[4] G. Senjanović and R. N. Mohapatra, "Exact Left-Right Symmetry and Spontaneous Violation of Parity," Phys.Rev. D12 (1975) 1502

[5] G. Senjanović, "Spontaneous Breakdown of Parity in a Class of Gauge Theories," Nucl.Phys. B153 (1979) 334-364

[6] R. N. Mohapatra and G. Senjanović, "Neutrino Mass and Spontaneous Parity Violation,"
Phys.Rev.Lett. 44 (1980) 912.

[7] R. N. Mohapatra and G. Senjanovic, "Neutrino Masses and Mixings in Gauge Models with Spontaneous Parity Violation," Phys. Rev. D23 (1981) 165.

[8] J. C. Pati and A. Salam, "Unified Lepton-Hadron Symmetry and a Gauge Theory of the Basic Interactions," Phys. Rev. D8 (1973) 1240-1251

[9] J. C. Pati and A. Salam, "Are There Anomalous Lepton-Hadron Interactions?," Phys. Rev. Lett. 32 (1974) 1083

[10] N. G. Deshpande, J. F. Gunion, B. Kayser, and F. I. Olness, "Left-right symmetric electroweak models with triplet Higgs," Phys. Rev. D44 (1991) 837-858.

[11] J. Heeck and S. Patra, "Minimal Left-Right Symmetric Dark Matter," 
Phys. Rev. Lett. 115 (2015) no. 12, 121804 arXiv:1507.01584

[12] C. Garcia-Cely and J. Heeck, "Phenomenology of left-right symmetric dark matter," arXiv:1512.03332

[13] P. S. Bhupal Dev and R. N. Mohapatra, "Unified explanation of the eejj, diboson and dijet resonances at the LHC," Phys. Rev. Lett. 115 (2015) no. 18, 181803. arXiv: 1508.02277

[14] F. F. Deppisch, L. Graf, S. Kulkarni, S. Patra, W. Rodejohann, N. Sahu, and U. Sarkar, "Reconciling the $2 \mathrm{TeV}$ Excesses at the LHC in a Linear Seesaw Left-Right Model," arXiv:1508.05940.

[15] S. Patra, F. S. Queiroz, and W. Rodejohann, "Stringent Dilepton Bounds on Left-Right Models using LHC data," Phys. Lett. B752 (2016) 186-190, arXiv:1506.03456

[16] F. F. Deppisch, T. E. Gonzalo, S. Patra, N. Sahu, and U. Sarkar, "Signal of Right-Handed Charged Gauge Bosons at the LHC?,"

Phys. Rev. D90 (2014) no. 5, 053014 arXiv: 1407.5384

[17] V. Tello, M. Nemevsek, F. Nesti, G. Senjanovic, and F. Vissani, "Left-Right Symmetry: from LHC to Neutrinoless Double Beta Decay," Phys. Rev. Lett. 106 (2011) 151801, arXiv:1011.3522

[18] S. Das, F. Deppisch, O. Kittel, and J. Valle, "Heavy Neutrinos and Lepton Flavour Violation in Left-Right Symmetric Models at the LHC," Phys.Rev. D86 (2012) 055006, arXiv:1206.0256

[19] J. Barry and W. Rodejohann, "Lepton number and flavour violation in TeV-scale left-right symmetric theories with large left-right mixing," JHEP 1309 (2013) 153, arXiv:1303.6324

[20] R. L. Awasthi, M. Parida, and S. Patra, "Neutrino masses, dominant neutrinoless double beta decay, and observable lepton flavor violation in left-right models and $S O(10)$ grand unification with low mass $W_{R}, Z_{R}$ bosons," JHEP 1308 (2013) 122, arXiv:1302.0672

[21] J. Chakrabortty, H. Z. Devi, S. Goswami, and S. Patra,
"Neutrinoless double- $\beta$ decay in TeV scale Left-Right symmetric models," JHEP 08 (2012) 008 , arXiv: 1204.2527

[22] B. A. Dobrescu and Z. Liu, "W Boson near 2 TeV: Predictions for Run 2 of the $L H C$," Phys. Rev. Lett. 115 (2015) no. 21, 211802 arXiv:1506.06736.

[23] J. Brehmer, J. Hewett, J. Kopp, T. Rizzo, and J. Tattersall, "Symmetry Restored in Dibosons at the LHC?," JHEP 10 (2015) 182, arXiv:1507.00013.

[24] P. Bhupal Dev, S. Goswami, M. Mitra, and W. Rodejohann, "Constraining Neutrino Mass from Neutrinoless Double Beta Decay," Phys.Rev. D88 (2013) 091301, arXiv:1305.0056

[25] C.-Y. Chen, P. S. B. Dev, and R. N. Mohapatra, "Probing Heavy-Light Neutrino Mixing in Left-Right Seesaw Models at the LHC," Phys. Rev. D88 (2013) 033014, arXiv:1306.2342

[26] C.-H. Lee, P. S. Bhupal Dev, and R. N. Mohapatra, "Natural TeV-scale left-right seesaw mechanism for neutrinos and experimental tests," Phys. Rev. D88 (2013) no. 9, 093010 arXiv: 1309.0774.

[27] X.-G. He and S. Rajpoot, "Anomaly Free Left-right Symmetric Models With Gauged Baryon and Lepton Numbers," Phys. Rev. D41 (1990) 1636

[28] M. Duerr, P. Fileviez Perez, and M. Lindner, "Left-Right Symmetric Theory with Light Sterile Neutrinos," Phys. Rev. D88 (2013) 051701, arXiv:1306.0568

[29] S. Patra and S. Rao, "Singlet fermion Dark Matter within Left-Right Model," arXiv:1512.04053.

[30] M. Duerr, P. Fileviez Perez, and M. B. Wise, "Gauge Theory for Baryon and Lepton Numbers with Leptoquarks," Phys. Rev. Lett. 110 (2013) 231801 arXiv: 1304.0576

[31] Planck, P. A. R. Ade et al., "Planck 2015 results. XIII. Cosmological parameters," arXiv:1502.01589. 